\title{
Trichostatin A enhances radiosensitivity and radiation-induced DNA damage of esophageal cancer cells
}

\author{
Shaobo Wang ${ }^{1 \#}$, Min Song ${ }^{2 \#}$, Bo Zhang ${ }^{1}$ \\ ${ }^{1}$ Department of Nephrology, Key Laboratory for the Prevention and Treatment of Chronic Kidney Disease of Chongqing, Chongqing Clinical \\ Research Center of Kidney and Urology Diseases, Xinqiao Hospital, Army Medical University (Third Military Medical University), Chongqing, \\ China; ${ }^{2}$ Department of Neurology, Second Affiliated Hospital of Chongqing Medical University, Chongqing, China \\ Contributions: (I) Conception and design: B Zhang; (II) Administrative support: B Zhang; (III) Provision of study materials or patients: S Wang; (IV) \\ Collection and assembly of data: S Wang, M Song; (V) Data analysis and interpretation: B Zhang; (VI) Manuscript writing: All authors; (VII) Final \\ approval of manuscript: All authors. \\ "These authors contributed equally to this work. \\ Correspondence to: Dr. Bo Zhang, MD. Department of Nephrology, Key Laboratory for the Prevention and Treatment of Chronic Kidney Disease \\ of Chongqing, Kidney Center of PLA, Xinqiao Hospital, Army Medical University (Third Military Medical University), 183 Xinqiao Street, \\ Chongqing, 400037, China. Email: bo_zhang@tmmu.edu.cn.
}

Background: Trichostatin A (TSA) is emerging as a potential component of anticancer therapy. In this study, we aimed to identify the radiosensitizing effects of TSA in esophageal squamous carcinoma cell lines and identify the genomic alteration of histone acetylation associated with TSA treatment.

Methods: EC109 and KYSE450 cells were pretreated with TSA $(0.1 \mu M)$ for 12 hours prior to irradiation, and the cell viability, flow cytometry, and comet assays were performed to analyze cell growth, cell apoptosis, and DNA damage, respectively. Chromatin immunoprecipitation sequencing (ChIP-Seq) was performed to identify the acetylation sites of histone $\mathrm{H} 3$ lysine 9 (H3K9), which was altered by TSA.

Results: Our data showed that TSA could sensitize esophageal cancer cells to radiation by inducing cell cycle arrest and increasing cell apoptosis. DNA damage induced by radiation was enhanced by TSA treatment. In addition, a total of 105 differential peak-related genes were found to be associated with TSA treatment, which was identified using ChIP-Seq with specific antibodies against acetylated histone H3K9.

Conclusions: Our data suggest that pretreatment with TSA can enhance ionizing radiation-induced DNA damage of esophageal cancer cells, which was associated with the altered histone modification of whole genome. TSA has potential implications for clinical use in increasing the anticancer efficacy of radiation.

Keywords: Trichostatin A (TSA); ionizing radiation; DNA damage; esophageal cancer cells

Submitted Aug 10, 2021. Accepted for publication Sep 29, 2021.

doi: 10.21037/jgo-21-560

View this article at: https://dx.doi.org/10.21037/jgo-21-560

\section{Introduction}

Esophageal cancer is one of the most aggressive cancers in the world. Esophageal squamous cell carcinoma (ESCC) is the dominant histological subtype in China $(1,2)$. The prognosis of ESCC in China is very poor, because it is frequently diagnosed at an advanced stage. Radiotherapy is one of the main treatments for clinical cancer therapy (3). Although much progress has been made during past decades, the side-effects of radiotherapy and inherent tumor radioresistance are still a major clinical challenge, which limits its clinical application. Therefore, new approaches to develop more effective strategies and to lower toxicity in clinical practice are urgently needed, and consequently a lot of research is currently trying to identify effective radiosensitizers.

Histone deacetylase (HDAC) deacetylates the lysine residues of both histones and non-histone targets $(4,5)$. 
Abnormal expression of HDACs in many cancers suggests that they may have a potential function in cancer genesis (6). Deregulation of HDACs can lead to the epigenetic modification of those oncogenes and cancer suppressors, thus HDACs might be a critical regulator of cancer genesis. Currently, HDACs is a new target for research on cancer treatments. Many HDAC inhibitors have been reported to increase the radiation lethality in many types of cancers. These findings suggest that HDAC inhibitors induce radiosensitization. Many HDAC inhibitors may have therapeutic potential to overcome radiation resistance of cancers (7). Trichostatin A (TSA), a pan-HDAC inhibitor, increases the radiosensitivity of non-small cell lung cancer, colon cancer, and glioblastomas cancer cells $(8,9)$. In our previous work, TSA enhanced the radiosensitivity of colon cancer cells, because apoptotic cell death induced by radiation was enhanced by TSA treatment (10). In addition, the radiosensitivity of esophageal cancer cells was enhanced by inhibition of HDAC1 expression via RNA interference (RNAi) (11). These findings indicate that both HDAC inhibitors and HDAC inhibition induce radiosensitization. HDAC inhibitors can reverse the epigenetic silencing that is frequently observed in cancer (12), but few studies have explored the whole alteration of histone acetylation. Therefore, it is important to identify the changes of histone acetylation sites in genomes. This will help to fully understand the functions and mechanisms of such inhibitors.

In this study, we aimed to identify the HDAC inhibitor TSA contributing to the radiosensitization of ESCC and its effects on the changes of histone acetylation sites by chromatin immunoprecipitation sequencing (ChIPSeq). We found that TSA promoted mitotic G2 gap 2 $(\mathrm{G} 2 / \mathrm{M})$ arrest, cell apoptosis, and DNA damage induced by radiation. Using ChIP-Seq analysis, TSA might directly participate in the DNA damage of esophageal cancer cells by decreasing the acetylation of histone $\mathrm{H} 3$ of growthrelated genes. These results indicate that TSA regulates key molecules involved in the growth pathway of esophageal cancer cells. We present the following article in accordance with the MDAR reporting checklist (available at https:// dx.doi.org/10.21037/jgo-21-560).

\section{Methods}

\section{Materials}

TSA, Cell Counting Kit-8 (CCK-8), and annexin-V staining kits were obtained from Beyotime Biotechnology Inc. (Nantong, China). Primary antibodies against acetylated histone H3 lysine 9 (H3K9), B-cell lymphoma 2 (Bcl-2), and poly adenosine diphosphate-ribose polymerase (PARP) were purchased from Cell Signaling Technology (Beverly, MA, USA). Anti-phosphorated H2A histone family member $\mathrm{X}(\mathrm{H} 2 \mathrm{AX})$ antibody was purchased from Cell Signaling (Billerica, MA, USA).

\section{Cell culture and irradiation}

Two esophageal cancer cell lines (EC109 and KYSE450) were obtained from the Cell Bank of the Chinese Academy of Sciences (Shanghai, China). The cells were cultured in Dulbecco modified Eagle medium (DMED, Hyclone, Logon, UT, USA) with $10 \%$ fetal bovine serum (FBS, Hyclone, Logon, UT, USA) at $37{ }^{\circ} \mathrm{C}$ with $5 \% \mathrm{CO}_{2}$. The cells were irradiated with 5 Gy of gamma irradiation using Cobalt-60 $\left({ }^{60} \mathrm{Co}\right) \mathrm{x}$-ray source. Then the culture medium was replaced.

\section{Cell viability assay}

The esophageal cancer cells were planted in 96-well plates with $8 \times 10^{3}$ cells per well. When the cells reached $50-70 \%$ confluence, the cells were pretreated with TSA $(0.1 \mu \mathrm{M})$ for $12 \mathrm{~h}$ and then treated with 5 Gy of $\gamma$-irradiation, according to our previous study (13). One hundred microliters of fresh medium and $10 \mu \mathrm{L}$ of CCK-8 (Dojindo, Kumamoto, Japan) were incubated together for $2 \mathrm{~h}$ and then detected using absorbance at $450 \mathrm{~nm}$. The cell viability assay was performed three times.

\section{Clone genesis assay}

The cells were plated in six-well plates and pretreated with TSA $(0.1 \mu \mathrm{M})$ for $12 \mathrm{~h}$ and then irradiated at a dose of 5 Gy. The cells were further cultivated for 10 to 14 days after replacing the medium. Then the cells were fixed and stained with $0.5 \%$ crystal violet. The number of colonies which contained more than 50 cells was counted using a microscope.

\section{Cell cycle}

Cells were pretreated with TSA $(0.1 \mu \mathrm{M})$ for $12 \mathrm{~h}$, and then irradiated with 5 Gy. Twenty-four hours after irradiation, cells were collected, stained with propidine iodide (PI) 
staining buffer for 30 min and analyzed with FACSCalibur (Becton Dickinson, Franklin Lakes, NJ, USA) using Modfit software (Becton Dickinson, Franklin Lakes, NJ, USA).

\section{Cell apoptosis}

Apoptosis was determined using the Annexin V-FITC Apoptosis Detection Kit (BD Biosciences, \#556547, Franklin Lakes, NJ, USA) according to the manufacturer's protocol. Briefly, cells were trypsinized and washed twice with cold phosphate-buffered saline (PBS). Cells were then stained with fluorescein isothiocyanate (FITC) conjugated annexin $\mathrm{V}$ and PI for $25 \mathrm{~min}$ at room temperature. Then cells were analyzed using FACSCalibur using Cellquest software (Becton Dickinson, Franklin Lakes, NJ, USA).

\section{Western blotting}

Protein samples were isolated from treated cells or the normal control by using RIPA buffer (Beyotime, Nantong China), and the protein concentration was determined using the Bradford dye-binding assay with bovine serum albumin as the standard. Total protein (20 $\mu \mathrm{g}$ per lane) was resolved in $12 \%$ SDS-polyacrylamide gel and transferred onto a polyvinylidene fluoride (PVDF) membrane, followed by blocking in $3 \%$ milk for $30 \mathrm{~min}$ at room temperature. Then the blot was further detected using antibodies directed against PARP (1:1,000, Cell Signaling Technology, \#9542, Danvers, MA, USA), Bcl-2 (1:2,000, Cell Signaling Technology, \#3498, Danvers, MA, USA) and glyceraldehyde-3-phosphate dehydrogenase (GAPDH) (1:2,000, Cell Signaling Technology, \#2118, Danvers, MA, USA). The bands were visualized and analyzed using the ChemiDoc ${ }^{\mathrm{TM}}$ Touch Imaging system (BIO-RAD, Hercules, CA, USA). GAPDH was used for normalization.

\section{Immunofuorescence staining for $\mathrm{H} 2 \mathrm{AX}$ foci}

Esophageal cancer cells were grown on chamber slides for $24 \mathrm{~h}$. After radiation, the cells were fixed and permeabilized with buffer containing 2\% paraformaldehyde and $0.2 \%$ Triton X-100 (Sigma Aldrich, St. Louis, MO, USA). Then the cells were blocked using $5 \%$ goat serum followed by hydride with anti-phospho-histone H2AX antibody. After staining, the cells were observed under a fluorescent microscope (Olympus, Tokyo, Japan).

\section{Comet assay}

The cancer cell suspension was embedded in a $0.7 \%$ lowmelting-point agarose. Then the suspension was spread on frosted slides covered with a $1 \%$ basal agarose layer. After solidification, the slides were immersed in lysing solution. The slides were washed three times using neutralization buffer to remove alkali and detergent. Then the slides were stained with ethidium bromide. The cells were observed under a fluorescent microscope (Olympus, Tokyo, Japan). DNA migration was analyzed by measuring the movement of the nuclear and the migrated DNA in 40 randomly chosen cells at each time point.

\section{ChIP-Seq}

The esophageal cancer cells were treated with formaldehyde to covalently crosslink proteins to DNA. Then sonication was performed to cut the chromatin into fragments of 100-300 bp. Immunoprecipitation with an antibody against acetylated histone $\mathrm{H} 3 \mathrm{~K} 9$ was carried out to enrich for acetylated histone $\mathrm{H} 3$-bound DNA compared to total chromatin. Then next generation sequencing was performed using Zhongkangbo Biotechnology (HiSeq 2500, Beijing, China). Briefly, independent DNA libraries were produced with the Illumina TrueSeq Library Kit (San Diego, CA, USA) as per manufacturer's instructions, and libraries were sequenced on the Illumina HiSeq producing $50 \mathrm{bp}$ single end reads. Then Bowtie2 (John Hopkins University (Baltimore, MD, USA) was used to align reads to the human genome (GRCh37/hg19), and peaks were called for each biological replicate using Model-based Analysis of ChIP-Seq (MACS2).

\section{Bioinformatics}

ChIP-Seq reads were mapped to the genome reference using software. Only the alignments within 2 mismatches were considered in peak calling. Whole genome peak scanning was based on a defined analysis model. Differential peaks were obtained using MAnorm software. After peak scanning, all the related genes were listed, and gene ontology was performed using GREAT (http://meme.sdsc. edu/). Kyoto Encyclopedia of Genes and Genomes (KEGG, a major public pathway-related database) was used in our pipeline to perform pathway enrichment analysis of peakrelated genes. 

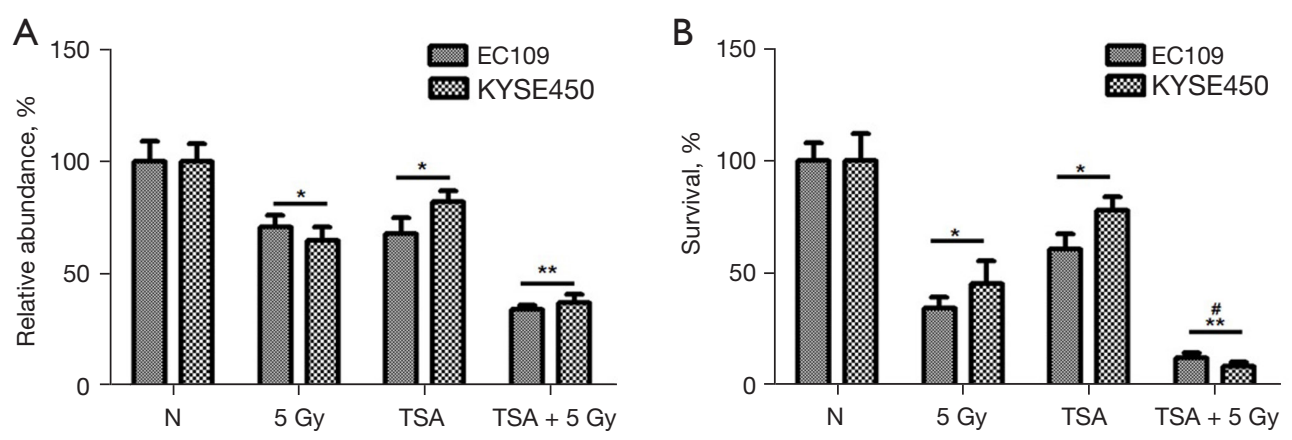

Figure 1 Radiosensitivity of esophageal squamous cell carcinoma was enhanced by TSA. (A) EC109 and KYSE450 cells were pretreated with TSA for 12 h, and then irradiated with 5 Gy. Cell viability was determined using Cell Counting Kit-8 assay. (B) EC109 and KYSE450 cells were seeded in 6-well plates and treated with TSA, and then irradiated with or without 5 Gy. Long-term survival was detected using clonogenic assay. $\mathrm{N}$, untreated control. *, $\mathrm{P}<0.05$; **, $\mathrm{P}<0.05$ vs. $\mathrm{N}$;, $\mathrm{P}<0.05$ vs. 5 Gy or TSA. TSA, trichostatin A; N, untreated control.

\section{Statistics}

Means, standard errors, and $\mathrm{P}$ values were calculated using SPSS version 20.0 for Windows (SPSS Inc., Chicago, IL, USA). Statistical analysis was performed using oneway analysis of variance for multi-group comparisons. All $\mathrm{P}$ values of less than 0.05 were considered as indicating statistical significance.

\section{Results}

\section{Enhancement of radiosensitivity induced by TSA}

Radiotherapy is an important approach to the treatment of ESCC. TSA is a classical histone deacetylase inhibitor (HDACi), which has pronounced antitumor activity. To investigate the effect of TSA on radiosensitivity, esophageal cancer cell lines of EC109 and KYSE450 were treated with radiation only or radiation combined with TSA, and cell viability was determined using the CCK- 8 assay. The cell viability of EC109 and KYSE450 decreased significantly when cells were treated with radiation and TSA (Figure 1A). To further confirm the effect of radiosensitivity of TSA, we performed a clonogenic assay to detect the long-term survival of esophageal cancer cells. Cell survival decreased significantly, when cells were treated with radiation and TSA (Figure $1 B$ and Figure S1). These results indicate that TSA sensitizes esophageal cancer cells to radiation.

\section{TSA promoted the G2/M arrest induced by radiation}

As radiation induces $\mathrm{G} 2 / \mathrm{M}$ arrest in many cancers, we wondered whether the radiosensitivity induced by TSA was due to cell cycle arrest. As expected, radiation induced G2/ $M$ arrest in both EC109 and KYSE450 cell lines (Figure 2). Moreover, cells in G2/M phase were increased when the cells were pretreated with TSA. These results indicated that TSA sensitizes esophageal cancer cells to radiation by cell cycle arrest.

\section{Apoptotic cell death induced by radiation was enbanced by TSA}

Cell apoptosis is a major type of cell death induced by radiation. We speculated whether decreased cell viability and clonogenic survival was due to cell apoptosis. To analyze the proportion of apoptotic cells, EC109 and KYSE450 were stained with annexin V-FITC and PI and then analyzed using flow cytometry. TSA increased apoptotic cells compared with cells that were not treated with TSA. Additionally, apoptotic cells were significantly increased by TSA and radiation treatment compared with cells treated only with radiation (Figure $3 A$ and Figure S2). In addition, the protein expression of apoptotic marker (cleaved PARP) in treated EC109 and KY SE450 cells was tested using Western blot. As demonstrated in Figure 3B, the proapoptotic form of PARP was increased in esophageal cancer cells treated with TSA and radiation, compared with those treated with radiation alone. These results indicate that TSA enhances cell apoptosis induced by radiation.

\section{TSA enhances DNA damage induced by radiation in esophageal cancer cells}

DNA damage is associated with radiation, and TSA 

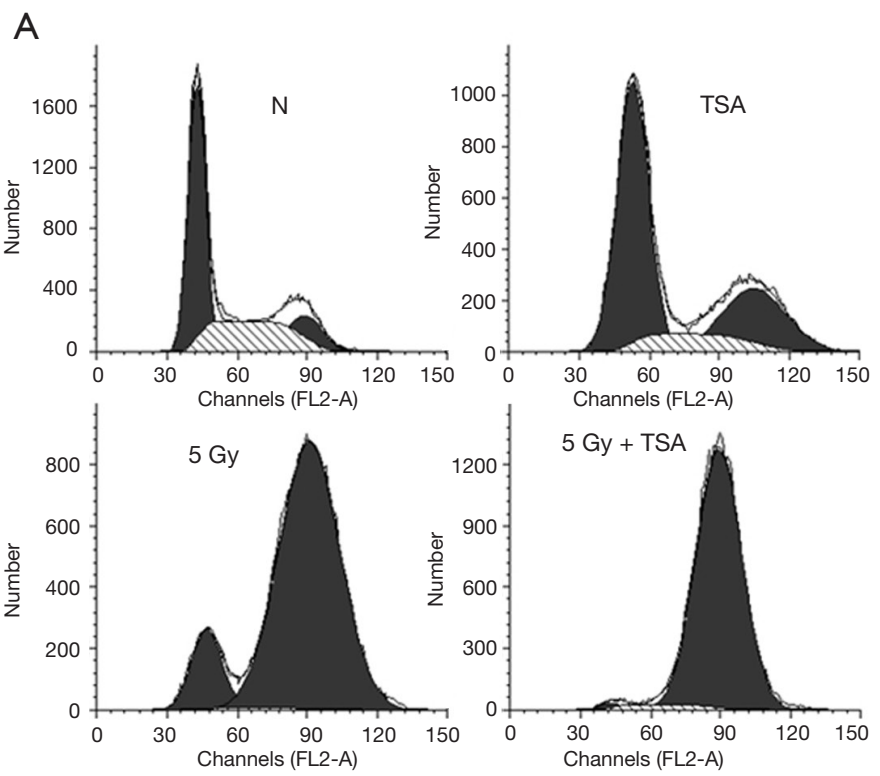

B

Figure 2 TSA promoted mitotic G2 gap 2 (G2/M) arrest induced by radiation. EC109 (A) and KYSE450 (B) cells were pretreated with TSA, followed by 5-Gy irradiation. Then cell cycle distributions were analyzed using flow cytometry. TSA, trichostatin A; N, untreated control.
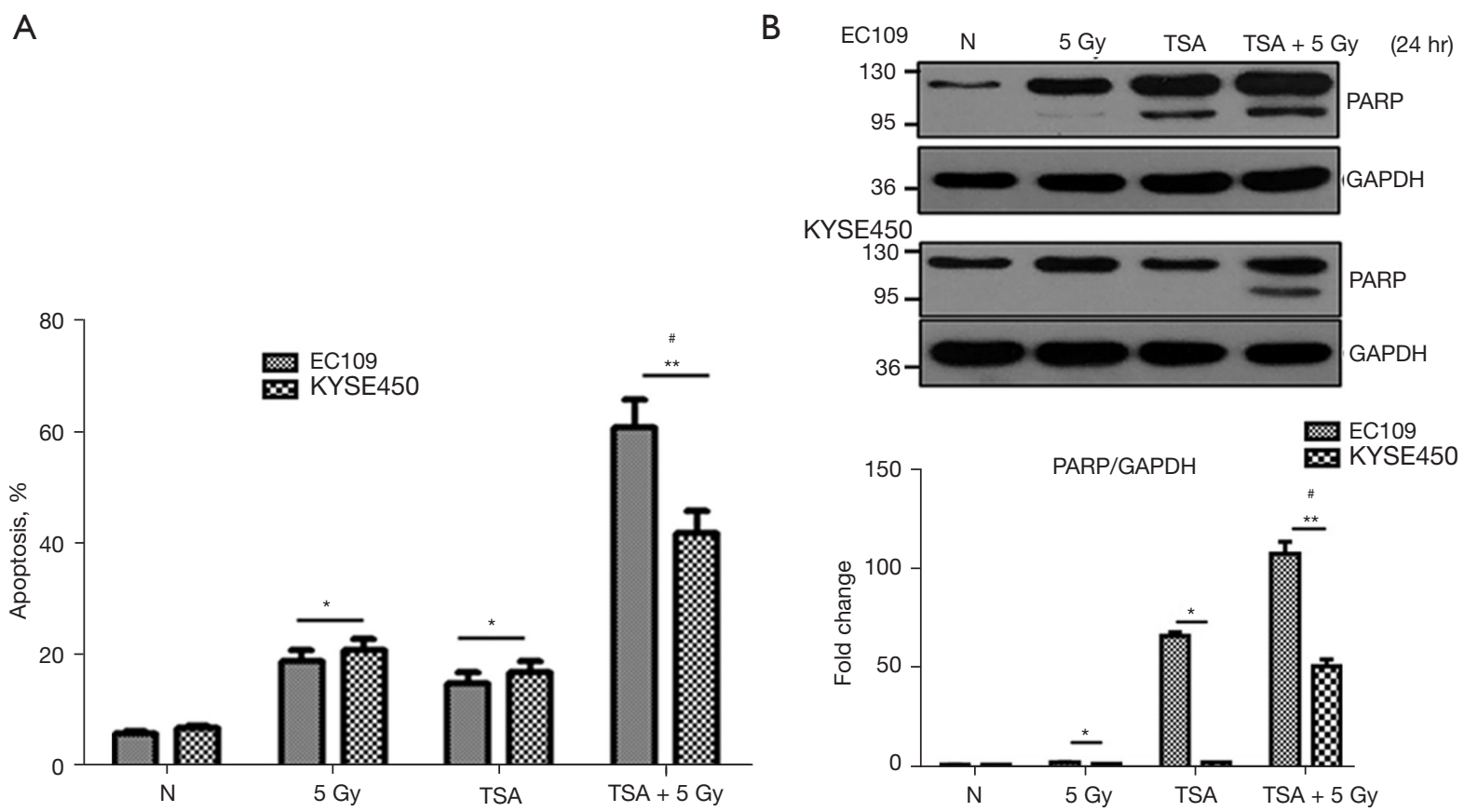

Figure 3 Apoptotic cell death induced by radiation was enhanced by TSA. (A) EC109 and KYSE450 cells were pretreated with TSA, followed by 5-Gy irradiation. Apoptotic cells were detected using staining with Annexin V-FITC Apoptosis Detection Kit and propidine iodide. (B) Protein samples from treated EC109 and KYSE450 cells were detected by western blot. Glyceraldehyde-3-phosphate dehydrogenase protein expression was measured as a control for equal loading. Fold changes of protein level were calculated by normalizing that of untreated control. *, $\mathrm{P}<0.05$; ${ }^{* *}, \mathrm{P}<0.01$ vs. N; ${ }^{*}, \mathrm{P}<0.05$ vs. 5 Gy or TSA. TSA, trichostatin A; N, untreated control. 
treatment results in changing the advanced chromatin structure. We speculated that DNA damage induced by radiation was enhanced by TSA treatment. Phosphorylation of $\mathrm{H} 2 \mathrm{AX}$ could form $\mathrm{cH} 2 \mathrm{AX}$ when double stranded (dsDNA) was broken. cH2 AX was detected using immunofluorescence after treatment with radiation and/or TSA. As shown in Figure 4A, TSA significantly enhanced the expression of radiation-induced $\mathrm{cH} 2 \mathrm{AX}$ foci in EC109 cells. Similar results were obtained from KYSE450 cells (Figure S3). In addition, DNA damage was analyzed using single-cell gel electrophoresis. Increased representative photomicrographs of tailed cells were seen in TSA treated cells (Figure 4B,4C). These results indicate that DNA damage induced by radiation is worsened by TSA treatment.

\section{TSA decreased the enrichment of acetylation histone $\mathrm{H} 3 \mathrm{~K} 9$ on growth-related genes}

As a HDAC inhibitor, TSA can disrupt the balance of acetylation and deacetylation of core histones, leading to changes of chromatin structure and subsequent gene expression. Next, we sought to determine which genes are regulated by TSA. To identify the changes of acetylated histone $\mathrm{H} 3$ induced by TSA genome-wide, we performed ChIP-Seq using a validated anti-Ac-histone H3K9 antibody. After peak calling, we identified 23,161 Ac-histone H3K9 peaks in EC109 cells. However, there are only 4,817 Achistone $\mathrm{H} 3 \mathrm{~K} 9$ peaks in TSA treated cells. In these peaks, the most frequent peaks are in the intergenic regions (Figure 5A). A total of 105 differential peak-related genes exhibited over two-fold enrichment in EC109-TSA vs. EC109-N (Table S1). To characterize the chromosome distribution of differential chromatin immunoprecipitation (ChIP) peaks, we counted the number of peaks on each chromosome (Figure 5B). Based on Gene Ontology (GO) analysis, we tried to find the potential functions of the peak-related genes. Gene ontology annotation showed that most of the 105 gene products participate in cellular processes and are located in cells with binding ability (Figure 5C, Tables 1 and 2). KEGG pathways analysis indicated that these genes are associated with metabolic pathways, pathways in cancer, endocytosis, protein digestion and absorption. The results indicated that TSA might directly participate in DNA damage of esophageal cancer cells by altering the acetylation of histone $\mathrm{H} 3$.

\section{Discussion}

HDACs are over-expressed in many cancers such as colorectal, gastric, lung, and breast cancer (14). In our previous study, we found than HDAC was over-expressed in ESCC. Therefore, HDAC inhibitor may be the therapy target for these cancers. HDAC inhibitors have various biological functions, such as transcriptional regulation, cell cycle arrest, cell apoptosis, and cell transformation. Recently, many HDAC inhibitors have been discovered, and TSA is one of the inhibitors that has the most potent deacetylase activity (15). Some researchers found that TSA has a potential function on radiosensitization of several types of cancer cells (16-18). In this study, we extended the radiosensitizing properties of TSA on human esophageal cancer cells. Using CCK-8 and clone genesis analysis, we found that TSA consistently radiosensitized both esophageal cancer cell lines. The level of radiosensitization might depend on the TSA concentration. There is another report of a similar concentration-dependent radiosensitization approach using TSA (19).

In addition to affecting hyperacetylation of core histones, TSA has been found to induce biological effects such as cell cycle arrest and cell apoptosis in a variety of cancer cell lines. Cell cycle and apoptosis are regarded as the major mechanisms underlying the anti-tumor activity of HDAC inhibitors (20). Our results indicate that the proportion of cells in the G2/M phase was significantly changed after treatment with TSA. The G2/M checkpoint is known as a determining factor of cellular radiosensitivity. We observed that pretreatment of TSA led to abrogation of G2/M phrase cells. The mechanism for the radiation-induced G2/M arrest using TSA is not fully understood. TSA also promotes the apoptosis of cancer cells by regulating the expression of apoptosis-related genes (21). Our study found that TSA increased the apoptosis induced by radiation. Using western blot, the cleaved PARP was found to be increased when the cells were pretreated with TSA. Another study found that TSA reduces the expression of Bcl-2 by mitochondrial channels, which results in an increase of the apoptosis of tumor cells (22).

It is well known that cellular radiosensitivity is related to DNA repair mechanisms (23-25). There is also some evidence that HDACs play a very important role in the repair process of DNA damage. DNA damage is more serious when TSA is used before the radiation of the cancer cells. The number of $\gamma-\mathrm{H} 2 \mathrm{AX}$ foci is significantly increased 


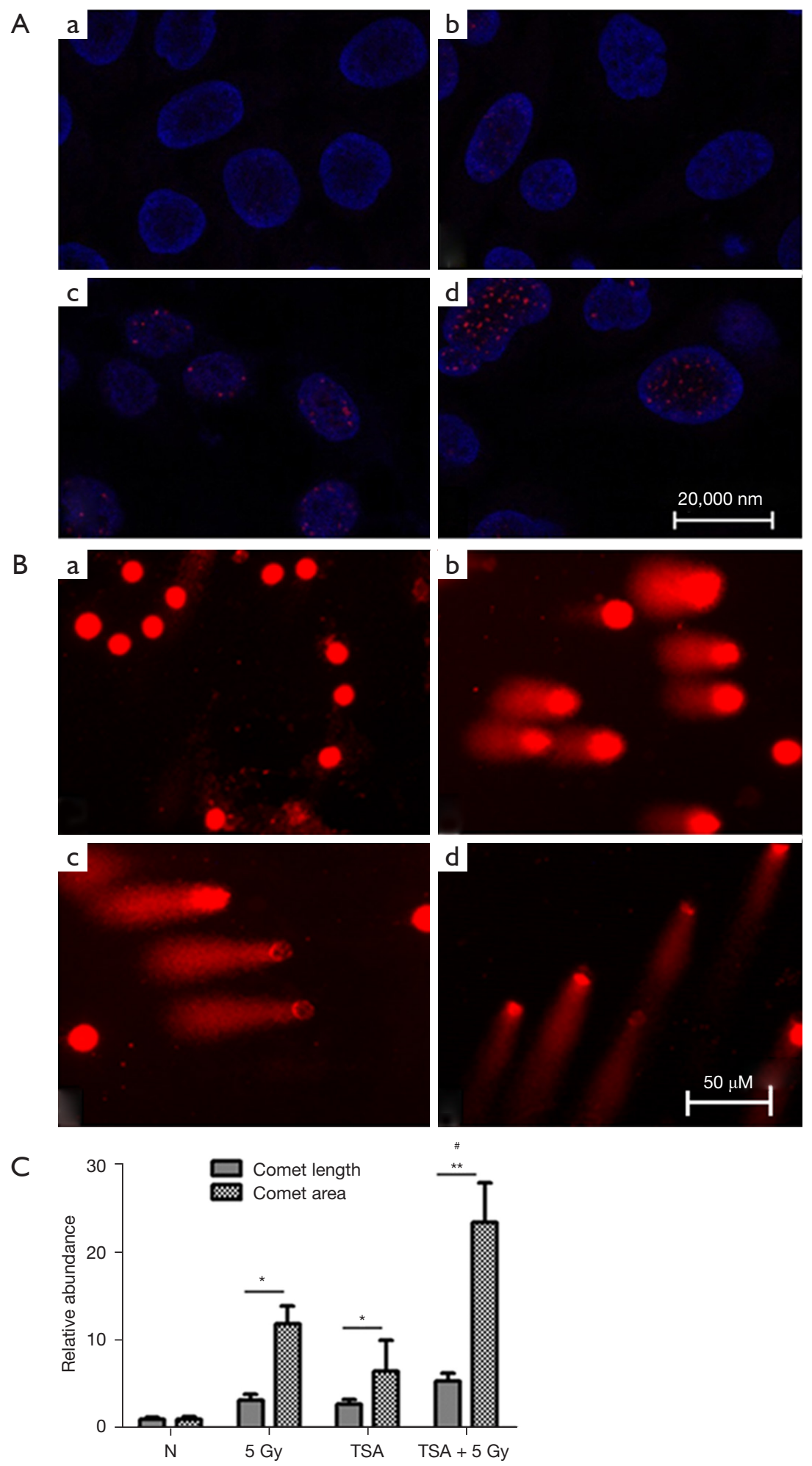

Figure 4 Radiation-induced DNA damage was enhanced using TSA in esophageal cancer cells. EC109 cells were pretreated with TSA, followed by 5-Gy irradiation. Then DNA damage was detected using immunofluorescent staining of $\gamma-\mathrm{H} 2 \mathrm{AX}$ (A) and single-cell gel electrophoresis (B,C). a: untreated control; b: TSA; c: 5 Gy; d: TSA + 5 Gy. *, $\mathrm{P}<0.05$; **, $\mathrm{P}<0.01$ vs. N; * $\mathrm{P}<0.05$ vs. 5 Gy or TSA. TSA, trichostatin A; N, untreated control. 
A

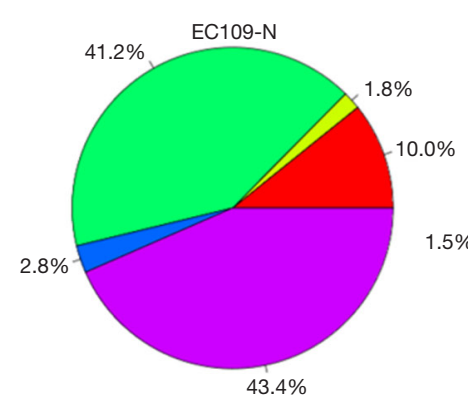

C

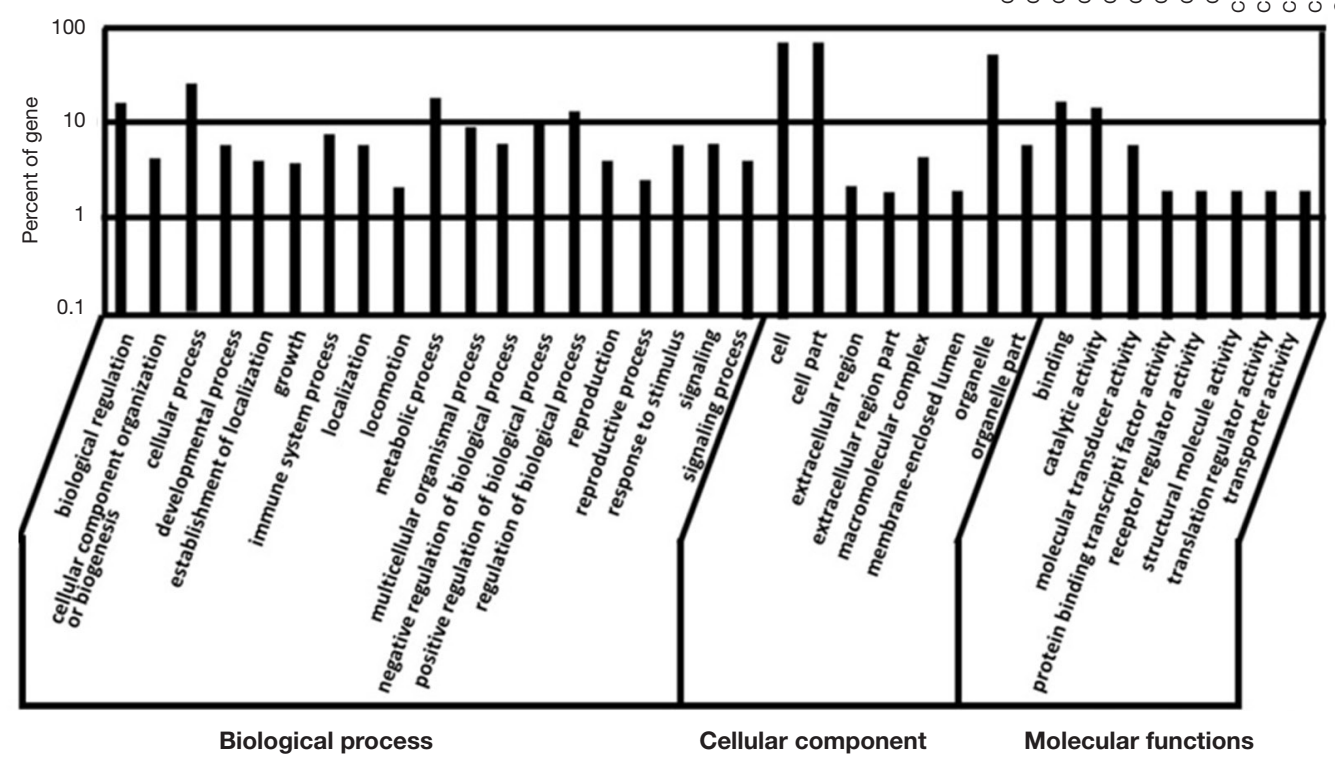

B

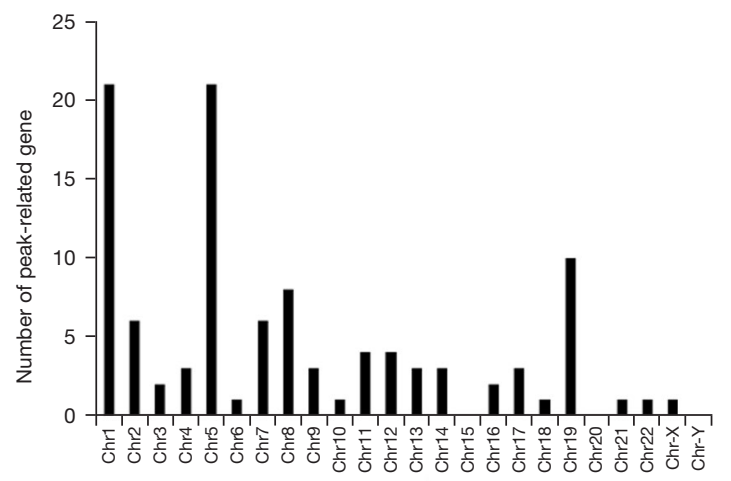

Figure 5 Acetylated histone H3K9 related DNA fragments were detected using ChIP-sequencing. EC109 cells were pretreated with TSA for $12 \mathrm{~h}$, then immunoprecipitation with an antibody against acetylated histone $\mathrm{H} 3 \mathrm{~K} 9$ was carried out to enrich for acetylated histone H3-bound DNA and subsequent sequencing. (A) The distribution of peaks in the genome was analyzed both in control EC109 or TSAtreated EC109 cells. (B) The number of differential chromatin immunoprecipitation (ChIP peaks) (EC109-TSA vs. EC109-N) on different chromosomes. (C) Gene Ontology annotation of differential peak-related genes in terms of biological process, cellular component, and molecular function. TSA, trichostatin A.

when TSA is added to the radiated cancer cells. $\gamma-\mathrm{H} 2 \mathrm{AX}$ foci represent the generation of the double-strand breaks (DSB) of DNA $(26,27)$. Smith $e t$ al. found that TSA induces DSB in HEK293T cells (28). These findings suggest that TSA enhances ionizing radiation-induced DNA damage in esophageal cancer cells. However, the precise mechanism of the proteins which potentiates radiosensitization by TSA needs to be defined.

As a HDACi, TSA enhances DNA damage by radiation in esophageal cancer cells. To identify the acetylated histone H3 genome-widely affected by TSA, we performed ChIPSeq using a validated $\mathrm{AcH} 3$ antibody. We found that a lot of genes are enriched with acetylation histone $\mathrm{H} 3 \mathrm{~K} 9$ in nonTSA treated cells, compared with those treated with TSA. The mechanism of HDAC inhibitors involved in DNA damage is different. RC307, which is an HDACi, enhances DNA damage of ovarian carcinoma cells when combined with an atypical retinoid (29). Another study found that TSA downregulates the expression of $\mathrm{Ku} 70$ and $\mathrm{Ku} 80$, and that DNA-dependent protein kinase catalytic subunits (DNA-PKcs) result in non-small cell lung cancer cells apoptosis (30).

Collectively, our results indicate that pretreatment with TSA enhances ionizing radiation-induced DNA damage 
Table 1 The biological process of GO annotation based on differential peaks associated gene

\begin{tabular}{llcc}
\hline GO_ID & \multicolumn{1}{c}{ Pathway name } & Number of genes & P value \\
\hline GO:0065007 & Biological regulation & 24 & 4.86 E04 \\
GO:0016043 & Cellular component organization or biogenesis & 5 & $2.38 E 03$ \\
GO:0009987 & Cellular process & 46 & $9.84 E 04$ \\
GO:0032502 & Developmental process & 6 & 4.26 E03 \\
GO:0051234 & Establishment of localization & 3 & $3.78 E 03$ \\
GO:0040007 & Growth & 4 & $4.68 E 02$ \\
GO:0002376 & Immune system process & 7 & 8.47 E03 \\
GO:0032501 & Multicellular organismal process & 4 & $1.29 E 04$ \\
GO:0048519 & Negative regulation of biological process & 6.57 E04 \\
GO:0050789 & Regulation of biological process & 23 & $7.46 E 04$ \\
GO:0050896 & Response to stimulus & 12 & $4.98 E 04$ \\
\hline
\end{tabular}

GO, Gene Ontology.

Table 2 Top 10 KEGG pathways based on differential peak-associated genes

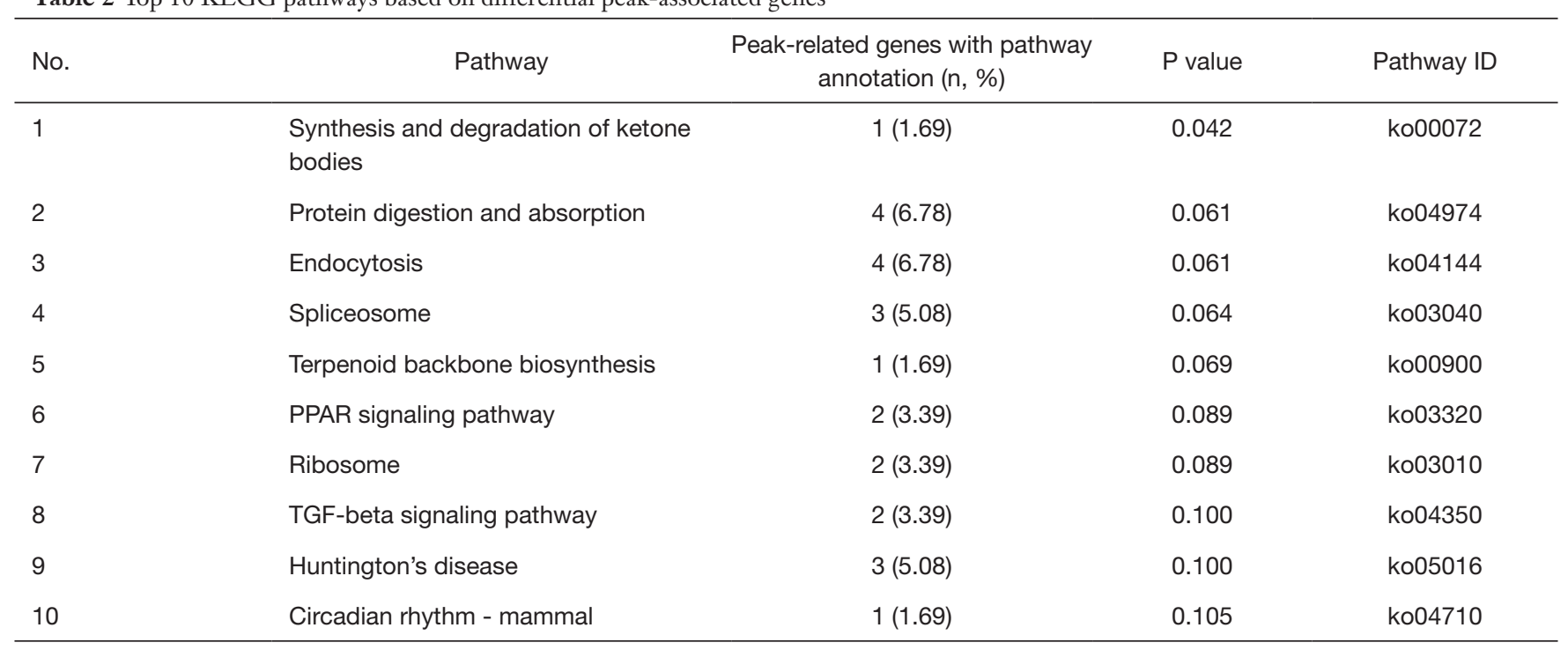

KEGG, Kyoto Encyclopedia of Genes and Genomes; PPAR, peroxisome proliferator-activated receptors; TGF, transforming growth factor.

of esophageal cancer cells. Therefore, TSA has potential implications for clinical use in increasing the anticancer efficacy of radiation.

\section{Acknowledgments}

Funding: This work was supported by a research grant from the National Natural Science Foundation of China (No. 81671331).

\section{Footnote}

Reporting Checklist: The authors have completed the MDAR reporting checklist. Available at https://dx.doi. org/10.21037/jgo-21-560

Data Sharing Statement: Available at https://dx.doi. org/10.21037/jgo-21-560

Conflicts of Interest: All authors have completed the ICMJE 
uniform disclosure form (available at https://dx.doi. org/10.21037/jgo-21-560). The authors have no conflicts of interest to declare.

Ethical Statement: The authors are accountable for all aspects of the work in ensuring that questions related to the accuracy or integrity of any part of the work are appropriately investigated and resolved. The study was conducted in accordance with the Declaration of Helsinki (as revised in 2013). Institutional ethical approval and informed consent were waived.

Open Access Statement: This is an Open Access article distributed in accordance with the Creative Commons Attribution-NonCommercial-NoDerivs 4.0 International License (CC BY-NC-ND 4.0), which permits the noncommercial replication and distribution of the article with the strict proviso that no changes or edits are made and the original work is properly cited (including links to both the formal publication through the relevant DOI and the license). See: https://creativecommons.org/licenses/by-nc-nd/4.0/.

\section{References}

1. Sun D, Cao M, Li H, et al. Cancer burden and trends in China: A review and comparison with Japan and South Korea. Chin J Cancer Res 2020;32:129-39.

2. Qiu ML, Lin JB, Li X, et al. Current state of esophageal cancer surgery in China: a national database analysis. BMC Cancer 2019;19:1064.

3. Lin HN, Chen LQ, Shang QX, et al. A meta-analysis on surgery with or without postoperative radiotherapy to treat squamous cell esophageal carcinoma. Int J Surg 2020;80:184-91.

4. Milazzo G, Mercatelli D, Di Muzio G, et al. Histone Deacetylases (HDACs): Evolution, Specificity, Role in Transcriptional Complexes, and Pharmacological Actionability. Genes (Basel) 2020;11:556.

5. Pant K, Peixoto E, Richard S, et al. Role of Histone Deacetylases in Carcinogenesis: Potential Role in Cholangiocarcinoma. Cells 2020;9:780.

6. Verza FA, Das U, Fachin AL, et al. Roles of Histone Deacetylases and Inhibitors in Anticancer Therapy. Cancers (Basel) 2020;12:1664.

7. Yao M, Rogers L, Suchowerska N, et al. Sensitization of prostate cancer to radiation therapy: Molecules and pathways to target. Radiother Oncol 2018;128:283-300.

8. Nagarajan D, Wang L, Zhao W, et al. Trichostatin A inhibits radiation-induced epithelial-to-mesenchymal transition in the alveolar epithelial cells. Oncotarget 2017;8:101745-59.

9. Wang X, Chen S, Shen T, et al. Trichostatin A reverses epithelial-mesenchymal transition and attenuates invasion and migration in MCF-7 breast cancer cells. Exp Ther Med 2020;19:1687-94.

10. He G, Wang Y, Pang X, et al. Inhibition of autophagy induced by TSA sensitizes colon cancer cell to radiation. Tumour Biol 2014;35:1003-11.

11. Song M, He G, Wang Y, et al. Lentivirus-mediated Knockdown of HDAC1 Uncovers Its Role in Esophageal Cancer Metastasis and Chemosensitivity. J Cancer 2016;7:1694-700.

12. Manzotti G, Torricelli F, Donati B, et al. HDACs control RUNX2 expression in cancer cells through redundant and cell context-dependent mechanisms. J Exp Clin Cancer Res 2019;38:346.

13. Liu Y, He G, Wang Y, et al. MCM-2 is a therapeutic target of Trichostatin A in colon cancer cells. Toxicol Lett 2013;221:23-30.

14. Bahl S, Seto E. Regulation of histone deacetylase activities and functions by phosphorylation and its physiological relevance. Cell Mol Life Sci 2021;78:427-45.

15. Sanaei M, Kavoosi F. Histone Deacetylases and Histone Deacetylase Inhibitors: Molecular Mechanisms of Action in Various Cancers. Adv Biomed Res 2019;8:63.

16. Kim MS, Baek JH, Chakravarty D, et al. Sensitization to $\mathrm{UV}$-induced apoptosis by the histone deacetylase inhibitor trichostatin A (TSA). Exp Cell Res 2005;306:94-102.

17. Artacho-Cordón F, Ríos-Arrabal S, Olivares-Urbano MA, et al. Valproic acid modulates radiation-enhanced matrix metalloproteinase activity and invasion of breast cancer cells. Int J Radiat Biol 2015;91:946-56.

18. Kuribayashi T, Ohara M, Sora S, et al. Scriptaid, a novel histone deacetylase inhibitor, enhances the response of human tumor cells to radiation. Int J Mol Med 2010;25:25-9.

19. Nagaraja SS, Krishnamoorthy V, Raviraj R, et al. Effect of Trichostatin A on radiation induced epithelialmesenchymal transition in A549 cells. Biochem Biophys Res Commun 2017;493:1534-41.

20. Usami M, Kikuchi S, Takada K, et al. FOXO3a Activation by HDAC Class IIa Inhibition Induces Cell Cycle Arrest in Pancreatic Cancer Cells. Pancreas 2020;49:135-42.

21. Sanaei M, Kavoosi F. Effect of Zebularine in Comparison to and in Combination with Trichostatin A on CIP/KIP Family (p21Cip1/Waf1/Sdi1, p27Kip1, and p57Kip2), 
DNMTs (DNMT1, DNMT3a, and DNMT3b), Class I HDACs (HDACs 1, 2, 3) and Class II HDACs (HDACs 4, 5, 6) Gene Expression, Cell Growth Inhibition and Apoptosis Induction in Colon Cancer LS 174T Cell Line. Asian Pac J Cancer Prev 2020;21:2131-9.

22. Song X, Wu JQ, Yu XF, et al. Trichostatin A inhibits proliferation of triple negative breast cancer cells by inducing cell cycle arrest and apoptosis. Neoplasma 2018;65:898-906.

23. Wang H, Wang B, Wei J, et al. Molecular mechanisms underlying increased radiosensitivity in human papillomavirus-associated oropharyngeal squamous cell carcinoma. Int J Biol Sci 2020;16:1035-43.

24. Hu C, Jiang X. The SUMO-specific protease family regulates cancer cell radiosensitivity. Biomed Pharmacother 2019;109:66-70.

25. Liu Y, Shen Y, Sun T, et al. Mechanisms regulating radiosensitivity of glioma stem cells. Neoplasma 2017;64:655-65.

26. Alipoor A, Fardid R, Sharifzadeh S. Evaluating Gamma$\mathrm{H} 2 \mathrm{AX}$ Expression as a Biomarker of DNA Damage

Cite this article as: Wang S, Song M, Zhang B. Trichostatin A enhances radiosensitivity and radiation-induced DNA damage of esophageal cancer cells. J Gastrointest Oncol 2021;12(5):19851995. doi: 10.21037/jgo-21-560 after X-ray in Angiography Patients. J Biomed Phys Eng 2018;8:393-402.

27. Khan K, Tewari S, Awasthi NP, et al. Flow cytometric detection of gamma-H2AX to evaluate DNA damage by low dose diagnostic irradiation. Med Hypotheses 2018;115:22-8.

28. Smith S, Fox J, Mejia M, et al. Histone deacetylase inhibitors selectively target homology dependent DNA repair defective cells and elevate non-homologous endjoining activity. PLoS One 2014;9:e87203.

29. Zuco V, Benedetti V, De Cesare M, et al. Sensitization of ovarian carcinoma cells to the atypical retinoid ST1926 by the histone deacetylase inhibitor, RC307: enhanced DNA damage response. Int J Cancer 2010;126:1246-55.

30. Robert C, Nagaria PK, Pawar N, et al. Histone deacetylase inhibitors decrease NHEJ both by acetylation of repair factors and trapping of PARP1 at DNA double-strand breaks in chromatin. Leuk Res 2016;45:14-23.

(English Language Editor: B. Meiser) 


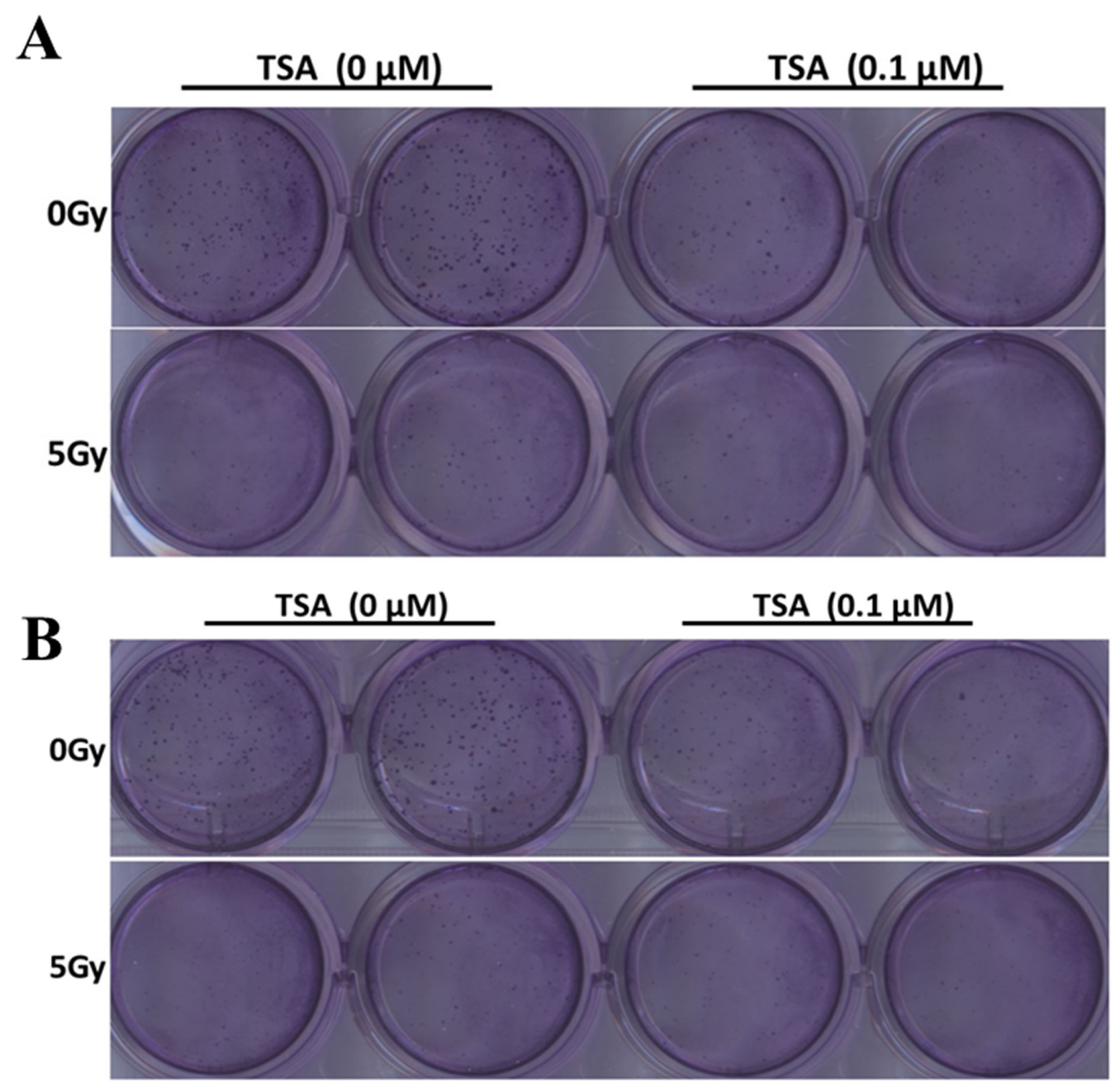

Figure S1 Long-term survival of esophageal cancer cells was analyzed by clone genesis assay. (A) EC109 cells were seeded into 6-well plates and pretreated with trichostatin A for $12 \mathrm{~h}$ and further treated with or without radiation. The cells were further cultivated for 10-14 days after replacing the medium. Then the cells were fixed and stained with $0.5 \%$ crystal violet. The number of colonies that included more than 50 cells was counted using a microscope. (B) KYSE450 were similarly treated as that of EC109 cells. 

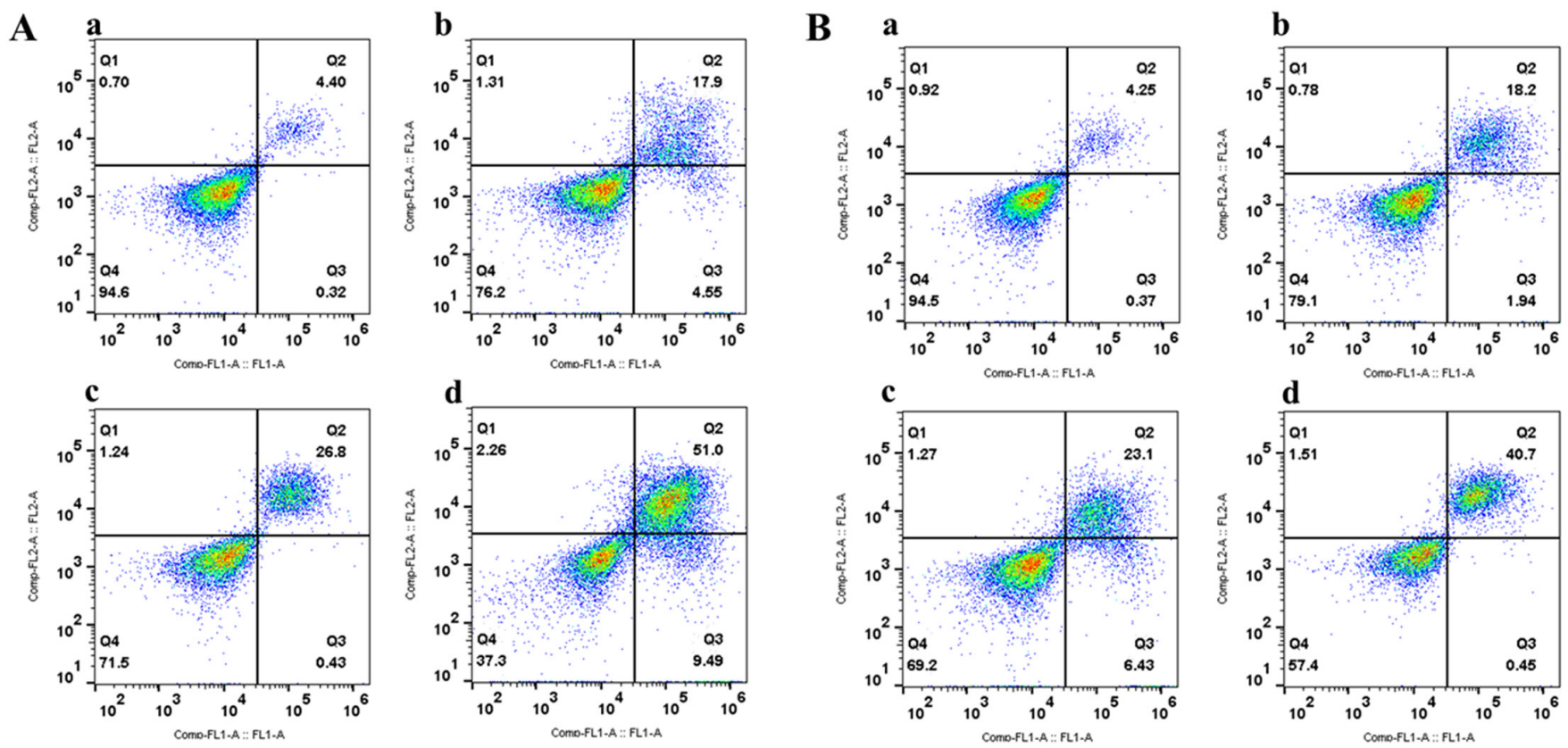

Figure S2 Representative images of apoptosis detection by flow cytometry. (A) EC109 cells were pretreated with trichostatin A (TSA) for 12 $\mathrm{h}$ and further treated with or without radiation. a: untreated control; b: TSA; c: 5 Gy; d: TSA + 5 Gy. (B) KYSE450 were similarly treated as that of EC109 cells. a: untreated control; b: TSA; c: 5 Gy; d: TSA + 5 Gy.
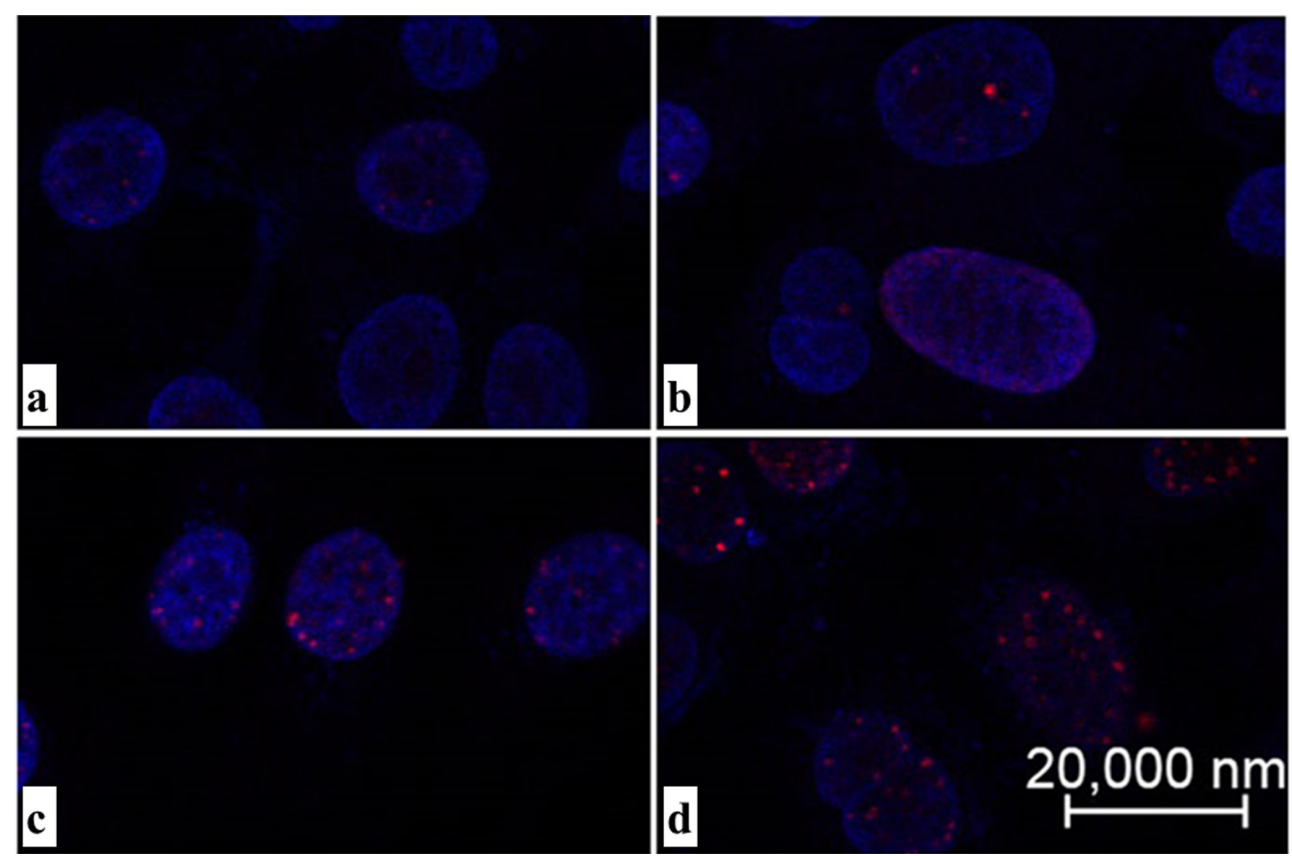

Figure S3 KYSE450 cells were pretreated with trichostatin A (TSA) for $12 \mathrm{~h}$ and further treated with or without radiation. Then the cells were fixed and permeabilized with buffer containing $2 \%$ paraformaldehyde and $0.2 \%$ Triton X-100. Then the cells were blocked using $5 \%$ goat serum, followed by hydride with anti-phospho-histone H2A histone family member X antibody. After staining with Cy3-conjugated secondary antibody, the cells were observed under a fluorescent microscope. (A) Untreated control; (B) TSA; (C) 5 Gy; (D) TSA + 5 Gy. 


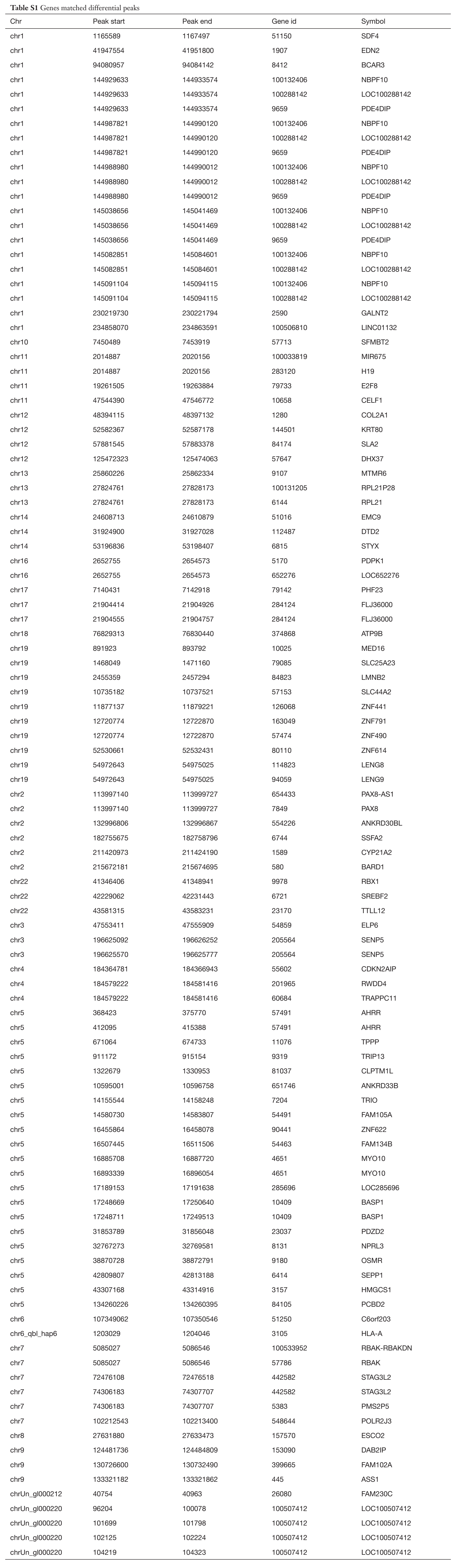

